\title{
CONTINUOUS MATRICES AND APPROXIMATE SIMILARITY
}

\author{
BY \\ JAMES C. LILLO( $\left.{ }^{1}\right)$
}

1. Introduction and notation. Let $M_{n}$ denote the set of all $n \times n$ matrices whose entries are bounded real valued continuous functions on the entire real line. Let $A, B \in M_{n}$. Then $A$ is said to be kinematically similar to $B$ (written $A \sim B)$ if there exists a $P(t)\left(P, P^{-1}, \dot{P} \in M_{n}\right)$ such that $B=P^{-1}(-\dot{P}+A P)$. It is clear that kinematic similarity defines a set of equivalence classes in $M_{n}$. For each $B \in M_{n}$ denote its equivalence class by $E(B)$. For $A \in M_{n}$ define $\eta[A]=\sum_{i, j=1}^{n}$ l.u.b.t $\left|a_{i j}(t)\right|$. For any subset $R$ of $M_{n}$ denote by $[R]-$ the closure of $R$ in the sense of the norm $\eta$. Let $A, B \in M_{n}$. Then $A$ is said to be approximately similar to $B$ (written $A \approx B$ ) if $B \in[E(A)]^{-}$. In an earlier paper [3] the author introduced the above concept and considered some of its properties and applications. In this paper we shall continue our study of this concept. In $\$ 2$ we introduce several invariants of approximate similarity and establish that it defines an equivalence relation in the set $K_{n}$, where $K_{n}$ denotes those matrices in $M_{n}$ all of whose entries are constant functions. Let $A_{n}$ denote the elements of $M_{n}$ all of whose entries are real valued almost periodic functions in the sense of Bohr. Define $L_{n}=\left\{B \mid B \in M_{n}\right.$ and $\left.\exists C \in K_{n} \ni B \approx C\right\}$. The set $A_{n} \cap L_{n}$ is of considerable importance with respect to applications to almost periodic systems of different equations [2]. It is known that this intersection contains open sets of the topology defined in $A_{n}$ by $\eta$ [4]. It is not known whether $A_{n} \cap L_{n}=A_{n}$. In $\S 3$ we obtain necessary and sufficient conditions that $A(t) \in A_{n}$ also belongs to $L_{n}$. Unfortunately the conditions obtained in $\$ 3$ depend upon the behavior of the solutions of the system $\dot{x}=A(t) x$. In $\S 4$ we obtain a sufficiency condition which depends more directly on the matrix $A(t)$ itself.

2. Invariants of approximate similarity. We shall first establish that the relation of approximate similarity defines an equivalence relation in the set $K_{n}$. We introduce the following notation. For $A \in K_{n}$ denote by $\lambda_{1}, \cdots, \lambda_{n}$ the characteristic roots of $A$, i.e. the roots of the equation $\operatorname{det}[A-\lambda I]=0$. Here roots of multiplicity $j$ are repeated $j$ times. For any complex number $\lambda$, $\operatorname{Re}(\lambda)$ and $\operatorname{Im}(\lambda)$ shall denote its real and imaginary parts respectively.

Lemma 2.1. If $\lambda_{1}, \cdots, \lambda_{n}$ are the characteristic roots of $A \in K_{n}$ then $C=\operatorname{diag}\left(\operatorname{Re}\left(\lambda_{1}, \cdots, \operatorname{Re}\left(\lambda_{n}\right)\right)\right.$ is approximately similar to $A$.

Proof. We must show that $A \in[E(C)]^{-}$. But $A \in[E(C)]^{-}$if and only if

Received by the editors October 10, 1961.

(1) This research was sponsored by N.S.F. grant G18915. 
$B \in[E(C)]^{-}$for any $B \in E(A)$. However, it is known that $E(A)$ contains a matrix $B$ of the form $\operatorname{diag}\left(B_{1}, \cdots, B_{k}\right)$. Here $B_{r}$ denotes an $m+1$ by $m+1$ square matrix of the form

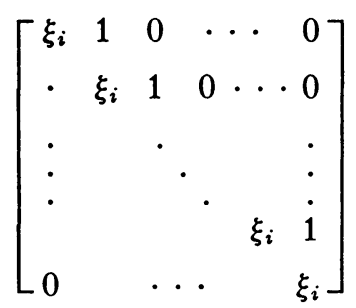

where $\xi_{i}=\operatorname{Re}\left(\lambda_{j}\right)=\operatorname{Re}\left(\lambda_{j+1}\right)=\cdots=\operatorname{Re}\left(\lambda_{j+m}\right)$. Thus if

$$
C_{r}=\operatorname{diag}\left(\operatorname{Re}\left(\lambda_{j}\right), \cdots, \operatorname{Re}\left(\lambda_{j+m}\right)\right)
$$

it suffices to show that for every $\epsilon>0$ there exists a $D_{r} \in E\left(C_{r}\right)$ such that $\eta\left[D_{r}-B_{r}\right]<\epsilon$. Assume that $2 q=m+1$ or $2 q=m$ and define $D^{\prime}=\left(d_{i j}\right)$ as follows:

$$
\begin{aligned}
d_{i j} & =b_{i j} & & \text { for all } i, j \text { such that } b_{i j} \neq 0, \\
d_{2 i, 2 i-1} & =(\epsilon / i)^{2} & & \text { for } i=1, \cdots, q, \epsilon<1, \\
d_{i j} & =0 & & \text { for all other } i, j .
\end{aligned}
$$

In the case $m+1=2 q, D_{r}$ possesses the distinct complex conjugate characteristic roots

$$
\xi_{i} \pm \frac{\epsilon}{j} \sqrt{-1}, \quad j=1, \cdots, q .
$$

In the case where $m=2 q, D_{r}$ also possesses the single real root $\xi_{i}$ in addition to the conjugate roots appearing in the first case. Thus in both cases the distinctness of the roots of $D_{r}$ assures us that $D_{r} \in E\left(C_{r}\right)$. This completes the proof of Lemma 2.1 .

Lemma 2.2. If $C=\operatorname{diag}\left[\operatorname{Re}\left(\lambda_{1}\right), \cdots, \operatorname{Re}\left(\lambda_{n}\right)\right]$ where $\lambda_{1}, \cdots, \lambda_{n}$ denote the characteristic roots of $A$ then $A \approx C$.

Proof. We must show that $C \in[E]-(A)$. As indicated in the proof of Lemma 2.1, however, it is known that $E(A)$ contains a matrix $B$ where $B$ is upper triangular (i.e. $b_{i j}=0$ for $i>j$ ) and the diagonal terms of $B$ agree with those of $C$. It then follows [3] that $C \in[E(B)]^{-}=[E(A)]^{-}$.

In general for $A$ and $B$ belonging to $M_{n}$ it is not known if $A \approx B$ implies $B \approx A$. The following theorem gives us this result for the case of the constant matrices.

THEOREM 2.1. If $A$ and $B \in K_{n}$ then $A \approx B$ and $B \approx A$ if and only if $\operatorname{Re}\left(\lambda_{i}\right)$ $=\operatorname{Re}\left(\sigma_{i}\right), i=1, \cdots, n$ where $\lambda_{i}$ and $\sigma_{i}$ are appropriate orderings of the characteristic roots of $A$ and $B$ respectively. 
Proof. In view of Lemmas 2.1 and 2.2 it will suffice to show that the real parts of the characteristic roots are common invariants for any two constant matrices $A$ and $B$ with $B \approx A$. It is well known that the roots of a polynomial are continuous functions of its coefficients. Thus for every $A$ and every $\epsilon>0$ there exists a $\delta>0$ such that $\eta\left[A-A^{\prime}\right]<\delta$ implies $\left|\operatorname{Re}\left(\sigma_{j}^{\prime}\right)-\operatorname{Re}\left(\lambda_{j}\right)\right|$ $\leqq\left|\sigma_{j}^{\prime}-\lambda_{j}\right|<\epsilon$ for $j=1, \cdots, n$ where $\sigma_{j}^{\prime}$ and $\lambda_{j}$ are appropriate orderings of the roots of $A^{\prime}$ and $A$ respectively. But if $B \approx A$ then for every $\delta>0$ there exists an $A^{\prime}$ such that $A^{\prime} \in E(B)$ and $\eta\left[A-A^{\prime}\right]<\delta$. Since $A^{\prime} \in E(B)$ we know that $\operatorname{Re}\left(\sigma_{j}^{\prime}\right)=\operatorname{Re}\left(\sigma_{j}\right), j=1, \cdots, n$, where $\sigma_{j}^{\prime}$ and $\sigma_{j}$ are appropriate orderings of the roots of $A^{\prime}$ and $B$. Thus $\operatorname{Re}\left(\sigma_{j}\right)=\operatorname{Re}\left(\lambda_{j}\right)$ for $j=1, \cdots, n$ and our result follows.

Since it is known [3] that the relation of approximate similarity is transitive and reflexive, as an immediate consequence of Theorem 1.1 we have the following corollary.

Corollary 2.1. Approximate similarity defines an equivalence relation in the set $K_{n}$.

The results of Markus [5] along with Corollary 2.1 assure us that approximate similarity defines an equivalence relation in the set of periodic matrices.

We now introduce for the set $M_{n}$ an invariant of approximate similarity which we will need in $\$ 3$. For this purpose we have the following definitions. Let $a(t)$ be a bounded continuous function for $-\infty<t<\infty$. Define the upper (lower) uniform mean value of $a(t)$, (written $M^{-}(a), M_{-}(a)$ ), to be

$$
\underset{T \rightarrow \infty}{\limsup } \underset{t_{0}}{\text { l.u.b. }} \frac{1}{T} \int_{t_{0}}^{t_{0}+T} a(\tau) d \tau\left(\lim _{T \rightarrow \infty} \underset{t_{0}}{\text { g.l.b. }} \frac{1}{T} \int_{t_{0}}^{t_{0}+T} a(\tau) d \tau\right) .
$$

Lemma 2.3. If $A \sim B$ then $M^{-}(\operatorname{trace} A)=M^{-}(\operatorname{trace} B)$ and $M_{-}(\operatorname{trace} A)$ $=M_{-}($trace $B)$.

Proof. Since $A \sim B$ there exists $P(t),\left(P(t), P^{-1}(t) \in M_{n}\right)$, such that if $\phi(t)$ is a fundamental matrix solution of the system $\dot{x}=A(t) x$ then $P(t) \phi(t)$ is a fundamental matrix solution of the system $\dot{y}=B(t) y$. Without loss of generality we may assume that $\operatorname{det} P(t)>0$ and $\operatorname{det} \phi(t)>0$. Thus we have that:

$$
\operatorname{det} \phi(t) / \operatorname{det} \phi(\tau)=\exp \int_{\tau}^{t} \operatorname{trace} A(s) d s
$$

and

$$
\operatorname{det} P(t) \operatorname{det} \phi(t) /[\operatorname{det} P(\tau) \operatorname{det} \phi(\tau)]=\exp \int_{\tau}^{t} \operatorname{trace} B(s) d s .
$$

From these two equalities we obtain 


$$
\int_{\tau}^{t} \operatorname{trace} A(s) d s-\int_{\tau}^{t} \operatorname{trace} B(s) d s=\ln [\operatorname{det} P(t)]+\ln \left[\operatorname{det} P^{-1}(\tau)\right] .
$$

Since $P(t)$ and $P^{-1}(t) \in M_{n}$ the desired result follows.

As an immediate result of the definition of approximate similarity and Lemma 2.3 we have the following theorem.

Theorem 2.2. If $A \approx B$ then $M^{-}($trace $A)=M^{-}($trace $B)$ and $M_{-}($trace $A)$ $=M_{-}(\operatorname{trace} B)$.

Corollary 2.2. If $B(t) \in M_{n}$ is approximately similar to $C \in K_{n}$ then $M^{-}(\operatorname{trace} B(t))=M_{-}(\operatorname{trace} B(t))$.

COROLlary 2.3. There exist elements in $M_{n}$ which are not in $L_{n}$.

If for any function $a(t)$ we have $M^{-}(a)=M_{-}(a)$ then we denote this common value by $M(a)$. We now have the following lemma.

LEMmA 2.4. If $M(a+b)=\lambda_{1}+\lambda_{2}$ and if $M^{-}(a) \leqq \lambda_{1}$ and $M^{-}(b) \leqq \lambda_{2}$ then $M(a)=\lambda_{1}$ and $M(b)=\lambda_{2}$.

Proof. Using the definition of $M, M_{-}, M^{-}$we have:

$$
\lambda_{1}+\lambda_{2}=M(a+b) \leqq M^{-}(a)+M^{-}(b) \leqq \lambda_{1}+\lambda_{2} .
$$

From this it follows that $M^{-}(a)=\lambda_{1}$ and $M^{-}(b)=\lambda_{2}$. But $\lambda_{1}+\lambda_{2}=M_{-}(a+b)$ $\leqq M_{-}(a)+M^{-}(b) \leqq \lambda_{1}+\lambda_{2}$ and so $M_{-}(a)=\lambda_{1}=M^{-}(a)=M(a)$. In the same way one may obtain $M_{-}(b)=\lambda_{2}=M^{-}(b)=M(b)$.

3. The class $L_{r}$. In this section we shall establish necessary and sufficient conditions that $A(t) \in A_{n}$ belongs to $L_{n}$. Given an almost periodic matrix $A(t)$, the hull of $A(t)$, written $H(A)$, is the set of all elements of $A_{n}$ which are uniform limits of sequences $\left\{A\left(t+\tau_{i}\right)\right\}$ of translates of $A(t)$.

Lemma 3.1. If for $A(t) \in A_{n}$ and $B \in K_{n}$ we have $A(t) \approx B$ then for every $A^{*} \in H(A), A^{*} \approx B$.

Proof. Since $A(t) \approx B$ there exists a sequence $\left\{P_{i}(t)\right\}$ such that $P_{i}, P_{i}^{-1}$, $\dot{P}_{i} \in M_{n}$ and $\eta\left[-P_{i}^{-1}\left(\dot{P}_{i}-A P_{i}\right)-B\right]<1 / i, i=1,2, \cdots$. Let $M_{i}$ denote the $\max \left(\eta\left[P_{i}\right], \eta\left[P_{i}^{-1}\right]\right)$ and $\left\{\tau_{j}\right\}$ be such that $\lim _{j \rightarrow \infty}\left(\eta\left[A\left(t+\tau_{j}\right)-A^{*}(t)\right]\right)=0$. We may assume that the $\left\{\tau_{j}\right\}$ have been chosen in such a way that $\eta\left[A\left(t+\tau_{j}\right)-A^{*}(t)\right]<1 / j\left(2 M_{j}\right)$. Then

$$
\eta\left[-P_{j}^{-1}\left(t+\tau_{j}\right)\left\{\dot{P}_{j}\left(t+\tau_{j}\right)-A^{*}(t) P_{j}\left(t+\tau_{j}\right)\right\}-B\right]<2 / j
$$

for $j=1,2, \cdots$. This concludes the proof of Lemma 3.1 .

To facilitate the statement of the next lemma we introduce the following notation. If in the definition of $\eta[A]$ we restrict the range of $t$ to the interval $[0,+\infty)$ then we shall denote the resulting norm $\eta^{+}[A] . M^{+}$will have a similar meaning. 
Lemma 3.2. Let $A(t) \in A_{n}, B \in K_{n} ; P_{i}(t), P_{i}^{-1}(t), \dot{P}_{i}(t) \in M_{n} ;$ and $\dot{P}_{i}(t)$ uniformly continuous, be such that

$$
\eta^{+}\left[-P_{i}{ }^{1}\left(\dot{P}_{i}-A(t) P_{i}\right)-B\right]<1 / i, \quad i=1,2, \cdots
$$

Then $A(t) \approx B$.

Proof. Take a sequence $\left\{T_{j}\right\}$ such that $\lim _{j \rightarrow \infty} T_{j}=+\infty$ and $\lim _{j \rightarrow \infty} \eta\left[A\left(t+T_{j}\right)-A^{*}(t)\right]=0$ for some $A^{*} \in H(A)$. We may also assume that $P_{i}\left(t+T_{j}\right)$, for each $i$ and an appropriate subsequence of the $T_{j}$, converge uniformly on any finite interval to $P_{i}^{*}(t)$ which satisfies

$$
\eta\left[-P_{i}^{*-1}\left(\dot{P}_{i}^{*}-A^{*} P^{*}\right)-B\right]<1 / i \text {. }
$$

Thus $A^{*} \approx B$. Lemma 3.1 now states that $A(t) \approx B$.

Consider the system $\dot{x}=A(t) x$. For any solution $\varphi(t)$ of this system let $\|\varphi(t)\|$ denote the sum of the absolute values of the components of $\varphi(t)$. A subspace $S$ of solutions of the above system is said to have a uniform characteristic exponent $\sigma$ if for every $\epsilon>0$ there is an $M_{\epsilon}$ such that $\|\varphi(t)\| /\|\varphi(\tau)\|$ $\leqq M_{\epsilon} \exp [(\sigma+\epsilon)(t-\tau)]$ for every $\varphi(t) \in S$ and every $t \geqq \tau \geqq 0$. $A(t)$ is said to have property I with constants $\sigma_{1}<\sigma_{2}<\cdots<\sigma_{r}$ and integers $\rho_{0}=0<\rho_{1}$ $<\cdots<\rho_{n}=n$ if for every $\sigma_{i}, 1 \leqq i \leqq r$, there is a $\rho_{i}$ dimensional subspace $S_{i}$ for the system $\dot{x}=A(t) x$ which possesses a uniform characteristic exponent $\sigma_{i}$.

The following theorem is similar to results of Diliberto [1] and Markus [5] for the case of kinematic similarity.

Theorem 3.1. If $A \in A_{n}$ then a n.a.s.c. that $A \in L_{n}$ is that $A(t)$ possess property I with constants $\sigma_{1}, \cdots, \sigma_{r}, \rho_{1}, \cdots, \rho_{r}$ and $M($ trace $A)$ $=\sum_{i=1}^{r} \sigma_{i}\left(\rho_{i}-\rho_{i-1}\right)$.

Proof. Assume that $A \approx B$, where $B \in K_{n}$. In light of Theorem 2.2 and Corollary 2.2 we need only show that $A$ satisfies property I with the constants $\sigma_{1}, \cdots, \sigma_{r}, \rho_{1}, \cdots, \rho_{r}$. Here the $\sigma_{i}$ denote the real parts of the characteristic roots $\lambda_{j}$ of $B$ and $\rho_{i}$ denotes the number of $\lambda_{j}$ with real parts equal to or less than $\sigma_{i}$. But by Theorem 2.1 and transitivity we have that $A \approx \operatorname{diag}\left[\operatorname{Re}\left(\lambda_{1}\right), \cdots, \operatorname{Re}\left(\lambda_{n}\right)\right]$. The desired estimates now follow from known results.

Assume that $A(t)$ possesses property I. Let $\varphi_{1}(t), \cdots, \varphi_{n}(t)$ be $n$ independent solutions of $\dot{x}=A(t) x$ such that for each value of $j, 0 \leqq j \leqq r$ the first $\rho_{j}$ of the $\varphi_{i}(t)$ span the solution space $S_{j}$. Now using these vectors form an orthonormal set of vectors using the Gram-Schmidt procedure. Let $P(t)$ denote the matrix formed by using these orthonormal vectors as columns. We write $P(t)=$ G.S. $\left(\varphi_{1}(t), \cdots, \varphi_{n}(t)\right)$. Then $P(t)$ is just the classical Perron transformation and $P^{-1}(t)[A(t) P(t)-\dot{P}(t)]=B(t)$ where $B(t)$ is upper triangular. We first show that $M^{+}\left(b_{k k}\right)=\sigma_{j}$ for $\rho_{j-1}<k \leqq \rho_{j}, j=1, \cdots, r$. Let $\psi\left(t_{1}, t\right)$ denote the fundamental matrix solution of $\dot{y}=B(t) y$ which for $t=t_{1}$ 
has the value $\psi\left(t_{1}, t_{1}\right)=I$. Let $\gamma_{1}\left(t_{1}, t\right), \cdots, \gamma_{n}\left(t_{1}, t\right)$ denote the columns of $\psi\left(t_{1}, t\right)$. Now $P(t)$ carries the linear spaces $S_{j}$ into linear spaces $S_{j}^{*}$ with similar properties. This is due to the fact that $\gamma_{i}\left(t_{1}, t\right)=P^{-1} \sum_{s=1}^{i} d_{i_{8}} \varphi_{s}(t)$ where the $d_{i s}$ are fixed constants depending only on $t_{1}$ and $i_{1}$. Thus it follows that $\gamma_{i}\left(t_{1}, t\right)$ $\in S_{j}^{*}$ for all $i \leqq \rho_{j}$ and since $\sum_{s=1}^{i} d_{i s} \varphi_{s}(t) \in S_{j}$ the constant $M_{\epsilon}^{*}=\eta\left[P^{-1}\right] M_{\epsilon}$ is independent of the particular choice of $t_{1} \geqq 0$. Thus for each $i, 0 \leqq i \leqq \rho_{j}$ and $t_{1} \geqq 0$ we have

$$
\exp \left(\int_{t_{1}}^{t_{1}+\tau} b_{i i}(s) d s\right) \leqq\left\|\gamma_{i}\left(t_{1}, t_{1}+\tau\right)\right\| /\left\|\gamma_{i}\left(t_{1}\right)\right\| \leqq M_{\epsilon}^{*} \exp \left[\left(\sigma_{j}+\epsilon\right) \tau\right]
$$

Therefore it follows that $M^{-+}\left(b_{j j}(t)\right) \leqq \sigma_{i}$ for $\rho_{i-1}<j \leqq \rho_{i}$. But by Lemma 2.3 $M^{+}($trace $B)$ exists and is equal to $M^{+}($trace $A)=\sum_{i=1}^{r} \sigma_{i}\left(\rho_{i}-\rho_{i-1}\right)$. By repeated application of Lemma 2.4 we have that $M^{+}\left(b_{j j}(t)\right)=\sigma_{i}$ for $\rho_{i} \geqq j>\rho_{i-1}$. Since $A(t) \in A_{n}$ the entries of $A$ form a uniformly bounded equicontinuous family of functions. In this case it is known that the entries of $A(t+\tau)$, $P(t+\tau), P^{-1}(t+\tau)$, and $\dot{P}(t+\tau)$ for $0 \leqq \tau<\infty$ also form a uniformly bounded equicontinuous family of functions [6]. Thus we may select a sequence $\left\{T_{j}\right\}, \lim _{j \rightarrow \infty} T_{j}=+\infty$, such that $\lim _{j \rightarrow \infty} \eta\left[A\left(t+T_{j}\right)-A^{*}(t)\right]=0$ and the sequences $\left\{P\left(t+T_{j}\right)\right\},\left\{P^{-1}\left(t+T_{j}\right)\right\},\left\{\dot{P}\left(t+T_{j}\right)\right\},\left\{B\left(t+T_{j}\right)\right\}$ converge uniformly on finite intervals to $P^{*}(t), P^{-1 *}(t), P^{*}(t)$ and $B^{*}(t)$ respectively. Since $M^{+}\left(b_{j j}\right)=\sigma_{i}$ for $\rho_{i-1}<j<\rho_{i}$ it follows that $M\left(b_{j j}^{*}\right)=\sigma_{i}$ for $\rho_{i-1}<j \leqq \rho_{i}$. Thus [3] $B^{*}(t) \approx C=\left(c_{i j}\right)$ where $c_{i j}=0$ for $j \neq i$ and $c_{j j}=\sigma_{i}$ for $\rho_{i-1}<j \leqq \rho_{i}$. Since $A^{*} \in E(B)$ it then follows that $A^{*} \approx C$. Lemma 3.1 now assures us that $A \approx C$. This completes the proof of Theorem 3.1 .

To facilitate the statement of Corollary 3.1 we introduce the following notation. Let $A \in A_{n}$ be written $A=A_{0}+A_{p}$ where the entries of $A_{0}$ are the mean values of the corresponding entries of $A$. Denote the characteristic roots of $A_{0}$ by $\lambda_{1}, \cdots, \lambda_{n}$.

Corollary 3.1. Let $A_{0}$ commute with $A(t)$ and $\int_{0}^{t} A(s) d s$ for each $0 \leqq t<\infty$, then $A(t) \approx \operatorname{diag}\left[\operatorname{Re}\left(\lambda_{1}\right), \cdots, \operatorname{Re}\left(\lambda_{n}\right)\right]$.

Proof. The assumptions on $A_{0}$ imply that $A(t), A_{0}$, and $A_{p}(t)$ commute for all values of $t \geqq 0[5]$. Therefore, for $t \geqq t_{1} \geqq 0$,

$$
\exp \left[\int_{t_{1}}^{t} A(s) d s\right]=\exp \left[A_{0}(t-s)\right] \exp \left[\int_{t_{1}}^{t} A_{p}(s) d s\right]
$$

is a fundamental matrix solution. Since $A_{p}(s) \in A_{n}$ and has zero mean value for every $\epsilon>0$ there exists an $M_{\epsilon}$ such that

$$
\left\|\frac{1}{\gamma} \int_{t_{1}}^{t_{1}+\gamma} A_{p}(s) d s\right\|<M_{\epsilon}+\epsilon \gamma
$$

for all positive $t_{1}$ and $\gamma$. It now follows that property $I$ is satisfied. Since 
$A \in A_{n}$ the condition on the trace is also satisfied. This completes the proof of Corollary 3.1.

4. Limit periodic matrices. Unfortunately the conditions stated in $\$ 3$ which assure that a matrix $D(t) \in A_{n}$ also belongs to $L_{n}$ depend on the behavior of the solutions of the system $\dot{x}=D(t) x$. In this section we shall establish a sufficient condition for a matrix $D(t) \in M_{n}$ to belong to $L_{n}$ which is more directly related to the matrix $D(t)$. This result is contained in Theorem 4.1. Corollary 4.1 contains an application of this result to the case of limit periodic matrices.

From the results of $\$ 2$ we know that for every $H_{i}(t) \in L_{n}$ there is a constant matrix $A_{i}=\operatorname{diag}\left(\sigma_{1}, \cdots, \sigma_{n}\right)$ such that $H_{i}(t) \approx A_{i}$. Here the $\sigma_{j}$ are real numbers and $A_{i}$ is uniquely determined by $H_{i}(t)$ up to a reordering of the $\sigma_{j}$. Since $H_{i}(t) \approx A_{i}$, for every $\epsilon>0$ there exists a $P_{i}(\epsilon, t)$ such that $\eta\left[P_{i}^{-1}\left(H_{i} P_{i}-\dot{P}\right)-A_{i}\right]<\epsilon$.

THEOREM 4.1. Let $\left\{H_{i}(t)\right\}$ be a sequence of matrices in $L_{n}$ converging to a matrix $D \in M_{n}$. If there exists a sequence of matrices $P_{i}\left(\epsilon_{i}, t\right)$ as described above such that $\lim _{i \rightarrow \infty} \epsilon_{i}=0$ and $\lim _{i \rightarrow \infty}\left(\eta\left[D-H_{i}\right] \eta\left[P\left(\epsilon_{i}, t\right)\right] \eta\left[P^{-1}\left(\epsilon_{i}, t\right)\right]\right)=0$ then $D(t) \in L_{n}$.

Proof. We first observe that $\eta\left[A_{i}\right] \leqq n \eta\left[H_{i}\right]$ and so the set of all $A_{i}$ associated with the sequence $\left\{H_{i}\right\}$ is a compact set. Thus by proper choice of a subsequence we may assume that the sequence $\left\{A_{i}\right\}$ which is associated with the sequence $\left\{H_{i}(t)\right\}$ converges to a limit $A$ as $i \rightarrow \infty$. We now consider the identity

$P_{i}^{-1}\left[-\dot{P}_{i}+D P_{i}\right]=A+\left[A_{i}-A\right]+P_{i}^{-1}\left[D-H_{i}\right] P_{i}+P_{i}^{-1}\left[H_{i} P-\dot{P}\right]-A_{i}$.

As $i \rightarrow \infty$ the right hand sides of this identity tends to $A$. This completes the proof of Theorem 4.1 .

Corollary 4.1. Let $D(t)$ be a limit periodic matrix and $\left\{H_{i}(t)\right\}$ be a sequence of periodic matrices with periods $T_{i}$ converging to $D(t)$. Then, if $\lim _{i \rightarrow \infty}\left(\eta\left[D-H_{i}\right] \exp \left[\left\{(n+1) \eta\left(H_{i}\right)+n-1\right\} T_{i}\right]\right)=0, D(t) \in L_{n}$.

Proof. Since the $H_{i}(t)$ are periodic there exists a $P_{i}(t)$ which satisfies the equation $P_{i}=H_{i}(t) P_{i}+P_{i} B_{i}$. Here $B_{i}$ is the $A_{i}$ of the preceding theorem except that it may contain 1's directly above the diagonal and so we have $\eta\left[B_{i}\right] \leqq n \eta\left[H_{i}\right]+n-1$. Using this estimate for $\eta\left[B_{i}\right]$ we obtain:

$$
\eta\left[P_{i}\right] \leqq P(0) \exp \left[\left\{(n+1) \eta\left[H_{i}\right]+n-1\right\} T_{i} / 2\right] .
$$

Since $P_{i}^{-1}$ satisfies the equation $\dot{P}_{i}^{-1}=P^{-1} H_{i}+B_{i} P^{-1}$ the same estimate will hold for $\eta\left[P_{i}^{-1}\right]$ and the desired result follows. We note, without proof, that the quantity $\exp \left[\left\{(n+1) \eta\left[H_{i}\right]+n-1\right\} T_{i}\right]$ may be replaced by 


$$
\left(1+\sum_{j=1}^{n-1} \frac{1}{\gamma_{i}^{j}}\right) \exp \left[\left\{(n+1) \eta\left[H_{i}\right]+(n-1) \gamma_{i}\right\} T_{i}\right]
$$

where the $\gamma_{i}$ are any positive constants.

\section{BIBLIOGRAPHY}

1. S. Diliberto, On systems of ordinary differential equations. Contributions to the theory of nonlinear oscillations, pp. 1-39, Annals of Mathematics Studies, no. 20, Princeton Univ. Press, Princeton, N. J., 1950.

2. J. Lillo, Perturbations of nonlinear systems, Acta Math. 103 (1960), 123-138.

3. - Continuous matrices and the stability theory of differential systems, Math. Z. 73 (1960), 45-58.

4. - Approximate similarity and almost periodic matrices, Proc. Amer. Math. Soc. 12 (1961), 400-407.

5. L. Markus, Continuous matrices and the stability of differential systems, Math. Z. 62 (1955), 310-319.

6. W. T. Reid, Remarks on a matrix transformation for linear differential equations, Proc. Amer. Math. Soc. 8 (1957), 708-712.

Purdue University,

LAFAYETTE, INDIANA 